\author{
Ecología
}

\title{
Diversidad y estructura de un pastizal en El Cerrillo, Piedras Blancas, Estado de México, México
}

\author{
Diversity and structure of a grassland at El Cerrillo, Piedras Blancas, Estado de México, Mexico \\ Jonás Álvarez-Lopeztello $^{\mathrm{a}, *}$, Irma Victoria Rivas-Manzano ${ }^{\mathrm{b}}$, Luis Isaac Aguilera-Gómez ${ }^{\mathrm{b}}$ \\ y Manuel González-Ledesma ${ }^{\mathrm{c}}$ \\ ${ }^{a}$ Centro Interdisciplinario de Investigación para el Desarrollo Integral Regional, Instituto Politécnico Nacional, Hornos 1003, 71230 Santa Cruz Xoxoxcotlán, \\ Oaxaca, México

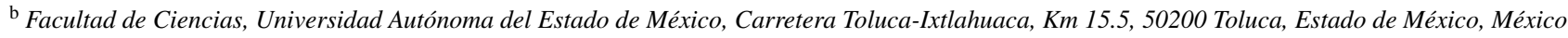 \\ ${ }^{\mathrm{c}}$ Centro de Investigaciones Biológicas, Universidad Autónoma del Estado de Hidalgo, Carretera Pachuca-Tulancingo, Km 4.5, 69000, Pachuca, \\ Hidalgo, México
}

Recibido el 6 de octubre de 2015; aceptado el 16 de marzo de 2016

Disponible en Internet el 9 de agosto de 2016

\begin{abstract}
Resumen
El conocimiento sobre la flora y la dinámica de la vegetación en los pastizales templados de México es hasta la fecha escaso. Con el fin de paliar esta deficiencia, en este estudio se evaluó la composición florística, diversidad y estructura de un pastizal templado del centro de México. Se registraron 118 especies, 93 géneros y 40 familias. Las familias mejor representadas a nivel genérico y específico son: Poaceae, Asteraceae, Fabaceae y Orchidaceae, esta última con una nueva especie aún no descrita perteneciente al género Cyclopogon. El estrato herbáceo del pastizal es complejo y cubre $98 \%$ de la superficie. Se encontró un porcentaje elevado de taxa nativos (20.50) y un índice de diversidad (Simpson y Shannon =3.36) relativamente alto. Muhlenbergia phalaroides fue la especie dominante y a ella correspondió el mayor valor de importancia. Los resultados sugieren que estos pastizales representan un reservorio considerable de especies relevantes para la conservación biológica, a pesar de estar sometidos a disturbios constantes.

Derechos Reservados (C 2016 Universidad Nacional Autónoma de México, Instituto de Biología. Este es un artículo de acceso abierto distribuido bajo los términos de la Licencia Creative Commons CC BY-NC-ND 4.0.
\end{abstract}

Palabras clave: Pastizales templados; Estructura de la vegetación; Conservación; Estrato herbáceo; Ecología de pastizales

\begin{abstract}
Current knowledge of the flora and vegetation dynamics in temperate grasslands of Mexico is still scarce. In order to mitigate this deficiency, in this study the floristic composition, diversity and structure of a temperate grassland of central Mexico were evaluated. A total of 118 species, 93 genera and 40 families were recorded. The best represented families, both at the generic and specific levels are: Poaceae, Asteraceae, Fabaceae and Orchidaceae. The latter including a new species belonging to the genus Cyclopogon. The herbaceous stratum of the grassland is complex, and covers $98 \%$ of the surface. A high percentage of native species was found (20.50) and a relatively high diversity index was calculated (Simpson and Shannon =3.36). Muhlenbergia phalaroides was the dominant species and it corresponds to the highest importance value. The results suggest that these grasslands represent a sizable reservoir of species relevant for biological conservation, despite being subjected to frequent disturbances. All Rights Reserved (C) 2016 Universidad Nacional Autónoma de México, Instituto de Biología. This is an open access item distributed under the Creative Commons CC License BY-NC-ND 4.0.
\end{abstract}

Keywords: Temperate grasslands; Vegetation structure; Conservation; Herbaceous stratum; Grasslands ecology

\footnotetext{
* Autor para correspondencia.

Correo electrónico: peto_810@hotmail.com (J. Álvarez-Lopeztello).

La revisión por pares es responsabilidad de la Universidad Nacional Autónoma de México.
} 


\section{Introducción}

Las comunidades donde la cubierta vegetal está dominada por gramíneas reciben el nombre de pastizales (Miranda y Hernández, 1963; Rzedowski, 1978). Este tipo de vegetación desempeña un papel crucial en el mantenimiento y funcionalidad del ecosistema, sobre todo en aquellos hábitats donde no pueden establecerse comunidades arbóreas debido a las limitaciones ambientales. Por ejemplo, los pastizales pueden evitar la erosión del suelo e incrementar las tasas de infiltración de agua (Cruz, 1969); asimismo, estas comunidades revisten importancia para la conservación y el mantenimiento de la biodiversidad aun cuando están sometidas a perturbaciones (Wilson, Peet, Dengler y Pärtel, 2012). En cuanto a su aprovechamiento, los pastizales constituyen el medio natural para el uso pecuario (Rzedowski, 1978), por lo que junto con los bosques templados son posiblemente uno de los ecosistemas más alterados del país (Challenger, 1998). A pesar de su importancia, este tipo de vegetación se encuentra entre los ecosistemas menos protegidos y mayormente amenazados (Conabio, 1998; Coupland, 1979). El conocimiento actual de la flora y la dinámica de la vegetación en estas comunidades es todavía fragmentario, debido en gran medida a que la mayoría de las investigaciones de carácter florístico y ecológico se han efectuado en las zonas semiáridas (Gentry, 1957; Hernández, 1964; Hernández, Tapia y Buller, 1957; Rivas-Manzano, 1984; Rzedowski, 1975; SuárezMartínez, 1999) y tropicales (López-Olmedo, Pérez-García y Meave, 2006; López-Olmedo, Meave y Pérez-García, 2007; Pérez-García y Meave, 2006; Reyes y Zamora, 1977).

Los pastizales han sufrido transformaciones intensas a causa de la influencia humana, como el fuego y el pastoreo, lo que dificulta establecer su origen. De hecho, se ha sugerido que estos dos factores afectan notablemente la riqueza, composición y estructura de la comunidad (Harrison, Inouye y Safford, 2003; Rivas-Manzano, 1988). También se ha propuesto que estas alteraciones promueven la invasión de especies exóticas (Hobbs y Huenneke, 1992), lo que podría causar el desplazamiento y pérdida de numerosas especies nativas a nivel local en zonas densamente pobladas.

En el Estado de México, que es uno de los más poblados del país, la mayor parte de la vegetación está alterada, lo cual es particularmente cierto para los bosques de pinoencino (González-Plata, 1998; Rivas-Manzano y Vibrans, 2000). Esta situación ha favorecido el establecimiento de pastizales de origen secundario con la intervención de fuegos frecuentes. Además, estos pastizales son utilizados para el forrajeo de animales domésticos. A causa de todos estos factores, muchos pastizales primarios pero intensamente perturbados pueden tener una fisonomía y una composición florística similar a la de algunos pastizales secundarios. Como sucede continuamente, las opiniones de los especialistas difieren en cuanto a la formación de estas comunidades vegetales (López-Olmedo et al., 2006, 2007; Miranda y Hernández, 1963; Pérez-García y Meave, 2006; Rzedowski, 1978). No obstante, cualquiera que pudiese ser su origen, un inventario florístico y la cuantificación de la importancia relativa de cada especie que forme parte del pastizal, es el primer paso para establecer estrategias de aprovechamiento, manejo, conservación y restauración.

\section{Materiales y métodos}

El estudio se realizó en la propiedad federal de telecomunicaciones denominada «El Cerrillo Piedras Blancas» $\left(19^{\circ} 25^{\prime}\right.$ y $19^{\circ} 26^{\prime} \mathrm{N}, 99^{\circ} 39^{\prime}$ y $99^{\circ} 40^{\prime} \mathrm{O}$, a una altitud de $2,593 \mathrm{~m}$ ), municipio de Toluca, Estado de México (fig. 1). A partir de la información registrada en la estación meteorológica «Nueva Santa Elena» (durante 11 años, de 1998-2008), en la Facultad de Ciencias Agrícolas, ubicada a $2 \mathrm{~km}$ del sitio de estudio, de la Universidad Autónoma del Estado de México (UAEMéx), se estimó una temperatura media del mes más frío de $2.4{ }^{\circ} \mathrm{C}$ (diciembre) y una temperatura media del mes más cálido de $11.5^{\circ} \mathrm{C}$ (junio), con temperatura media anual de $8.2^{\circ} \mathrm{C}$. La precipitación media anual fue de $738.6 \mathrm{~mm}$, con marcada estacionalidad; distinguiéndose una estación seca (noviembre-febrero) y una lluviosa (junio-septiembre). El clima de la región es $\mathrm{C}\left(\mathrm{w}_{2}\right)(\mathrm{w})$ b (i) g, es decir, templado subhúmedo con régimen de lluvias en verano, con oscilación térmica (García, 1973).

Los terrenos, con una superficie de $20 \mathrm{ha}$, presentan una pendiente de entre 2 y $6 \%$. Las rocas dominantes son de tipo volcánico y clástico. Los suelos son de tipo vertisol pélico $(\mathrm{Vp})$ y feozem háplico (Hh), y se caracterizan por ser muy compactos, arcillosos y masivos; asimismo, exhiben grietas anchas y profundas en la época de sequía y muestran una capa de tepetate entre 10 y $50 \mathrm{~cm}$ de profundidad, lo que constituye una limitante para el uso agrícola. En esta zona existen algunos cuerpos de agua intermitentes, bordos El Charco y La Cruz, cercanos al área de estudio y un cuerpo de agua persistente, la presa Antonio Alzate (Martínez y Hernández, 2000). Estos terrenos se encuentran continuamente sometidos a perturbaciones, tales como incendios, entre 1 y 3 veces por año, y pastoreo a lo largo de todo el año (Álvarez-Lopeztello, 2010).

Durante un año, de julio de 2007 a agosto de 2008, se hicieron prospecciones quincenales a través de recorridos, donde se recolectaron todas las especies de plantas presentes. Posteriormente se determinaron con ayuda de literatura especializada (Dávila et al., 2006; Espinosa-García y Sarukhán, 1997; Rzedowski y Rzedowski, 2005). La nomenclatura taxonómica de las familias sigue a «The Plant List» (www.theplantlist.org) y el nombre científico de las especies y los autores siguen a «Tropicos» (www.tropicos.org). Para determinar las especies introducidas y su posible origen se consultó a Rzedowski y Calderón (1990) y a Villaseñor y Espinosa-García (2004). Los especímenes de respaldo se depositaron en el herbario de la Facultad de Ciencias de la UAEMéx.

La vegetación se evaluó con el método de línea de Canfield (Canfield, 1941). Se trazaron tres líneas paralelas de $100 \mathrm{~m}$ de longitud, separadas por $20 \mathrm{~m}$ entre cada una, a lo largo de una pendiente que desemboca en un cuerpo de agua temporal (fig. 1C). La vegetación fue estimada a lo largo de las líneas a intervalos de $10 \mathrm{~m}$, a fin de totalizar 5 sitios o unidades muestrales analizadas por línea (0-10, 20-30, 40-50, 60-70 y 90-100 m), las cuales sirvieron de base para el análisis general del pastizal 


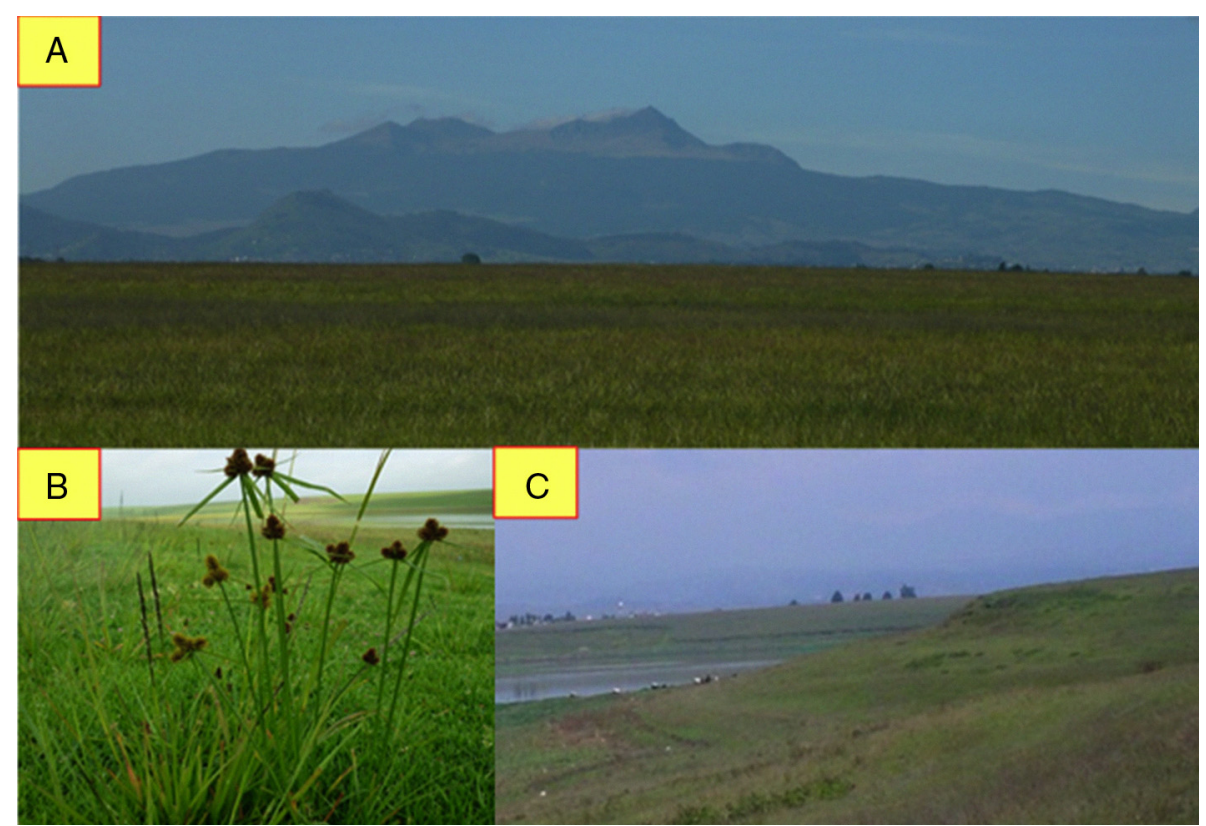

Figura 1. A) Vista del pastizal a nivel de paisaje, con el «Nevado de Toluca» al fondo. B) Cobertura del pastizal con Cyperus sp. al frente. C) Pendiente donde se realizó el muestreo de la vegetación. Fotografías: Ednita Espinoza Hernández (A) y Ana Mejía Canales (B).

y para explorar tanto la posible existencia de un gradiente de humedad como la respuesta de las especies a tal gradiente, por lo que las líneas se trazaron de la parte más alta de la pendiente a la más baja (fig. 1C). Se consideraron como sitios diferentes las unidades muestrales que variaron en la distancia al cuerpo de agua. Para el análisis se asignó el número 1 a los sitios más secos y el 5 a los más húmedos. Cada individuo interceptado por la línea fue incluido en el análisis, en especies amacolladas, se consideró a cada macollo como un solo individuo, y se les midió la altura y la cobertura lineal $(\mathrm{cm})$. El muestreo de la vegetación se llevó a cabo a finales de agosto e inicios de octubre de 2008.

El análisis de diversidad se evaluó mediante el índice de Simpson $\left(d_{s}\right)$ al igual que la dominancia $(\lambda)$ y la equidad $\left(e_{s}\right)$, adicionalmente se calculó el índice de Shannon-Wiener. La similitud florística entre los distintos sitios muestreados se examinó con los índices de similitud de Morisita y de Jaccard (Moreno, 2001). El análisis estructural incluyó el cálculo de la densidad, cobertura, frecuencia (valores absolutos y relativos), y posteriormente el cálculo del valor de importancia relativo (VIR; suma de los valores relativos) para cada especie presente. Se identificaron las especies dominantes, comunes, raras e indicadoras en el pastizal utilizando sus frecuencias y las abundancias relativas (Sokal y Rohlf, 1979). Se comparó la composición florística entre el pastizal y otras comunidades dominadas por gramíneas a diferentes niveles taxonómicos a partir del cálculo del índice de Sørensen.

\section{Resultados}

La lista florística del pastizal incluye 118 especies, distribuidas en 93 géneros y 40 familias de Angiospermas. Se distribuyen en 2 clases de plantas vasculares: (1) monocotiledóneas con 54 especies (45.8\%), las cuales incluyen una nueva especie aún no descrita perteneciente al género Cyclopogon
(Orchidaceae), además de 39 géneros $(41.9 \%)$ y 12 familias (30\%), y (2) eudicotiledóneas con 64 especies (54.2\%), 54 géneros $(58.1 \%)$ y 28 familias (70\%).

Las familias mejor representadas en cuanto al número de especies son: Poaceae con 25 especies (21.18\%), Asteraceae con $15(12.71 \%)$, Fabaceae y Cyperaceae con 7 (5.93\%) cada una, Orchidaceae 5 (4.24\%), e Iridaceae y Plantaginaceae, con $4(3.38 \%)$ cada una. Estas mismas familias poseen la mayor riqueza de géneros, Poaceae con 18 (19.35\%), Asteraceae con 13 (13.68\%), Fabaceae con 5 (5.26\%) y Cyperaceae y Orchidaceae con $4(4.21 \%)$ cada una, e Iridaceae y Plantaginaceae con 3 $(3.15 \%)$ cada una.

Los géneros más ricos en especies son: Muhlenbergia con 4 especies, Cyperus, Eragrostis, Sisyrinchium y Cologania, con 3 especies cada uno; en estos 5 géneros se concentra $13.55 \%$ de las especies (apéndice). De las especies presentes en el pastizal, $14(11.86 \%)$ son taxa introducidos a México, de los cuales 6 especies son originarias de África, 4 de Eurasia, 3 de Europa, 1 de Sudamérica y una especie de origen incierto (apéndice).

\section{Riqueza, diversidad y similitud florística}

El índice de diversidad de Simpson del pastizal alcanzó un valor general de 20.50; en contraste, el índice de ShannonWiener es de 3.36, mientras que la riqueza total es de 55 especies. Al analizar la vegetación en el gradiente de humedad, los índices de diversidad y la riqueza variaron considerablemente. Los sitios 2, 3 y 4 que son intermedios, tuvieron la misma riqueza específica y valores similares de diversidad y dominancia (tabla 1). Por el contrario, los sitios 1 y 5 que corresponden a los extremos del gradiente, donde 1 es el más seco y 5 el más húmedo, presentaron la menor riqueza específica, valores bajos de diversidad y valores altos de dominancia. La mayor similitud (Morisita) se obtuvo entre los sitios 1 y 2 , seguida por $\operatorname{los}$ sitios 3 y 4 ; en 
Tabla 1

Índices de diversidad, dominancia y equidad de Simpson y de Shannon-Wiener. Riqueza específica y número de individuos para el pastizal y cada uno de los sitios analizados.

\begin{tabular}{|c|c|c|c|c|c|c|}
\hline & \multicolumn{3}{|c|}{ Índice de Simpson } & \multirow[t]{2}{*}{ Índice de Shannon-Wiener } & \multirow[b]{2}{*}{$\begin{array}{l}\text { Riqueza } \\
\text { (S) }\end{array}$} & \multirow[b]{2}{*}{$\begin{array}{l}\text { Núm. de ind. } \\
\text { (N) }\end{array}$} \\
\hline & $\begin{array}{l}\text { Diversidad } \\
\left(d_{s}\right)\end{array}$ & $\begin{array}{l}\text { Dominancia } \\
(\lambda)\end{array}$ & $\begin{array}{l}\text { Equidad } \\
\left(\mathrm{e}_{\mathrm{s}}\right)\end{array}$ & & & \\
\hline Pastizal & 20.5 & 0.05 & 0.34 & 3.36 & 55 & 1,183 \\
\hline Sitio 1 & 8.01 & 0.13 & 0.3 & 2.41 & 24 & 217 \\
\hline Sitio 2 & 17.91 & 0.06 & 0.44 & 3.13 & 35 & 252 \\
\hline Sitio 3 & 16.24 & 0.06 & 0.4 & 3.07 & 35 & 296 \\
\hline Sitio 4 & 21.98 & 0.05 & 0.55 & 3.21 & 35 & 281 \\
\hline Sitio 5 & 10.02 & 0.1 & 0.37 & 2.6 & 23 & 137 \\
\hline
\end{tabular}

Tabla 2

Similitud florística (índices de Morisita - primer valor-y de Jaccard -segundo-) y número de especies comunes (se muestran entre paréntesis) para los distintos sitios analizados en el pastizal.

\begin{tabular}{|c|c|c|c|c|c|}
\hline Sitios & \multicolumn{5}{|c|}{ Similitud de Morisita y Jaccard } \\
\hline Sitio 1 & - & 0.86 y $0.47(19)$ & 0.63 y $0.51(20)$ & 0.52 y 0.51 (19) & 0.14 y $0.31(10)$ \\
\hline Sitio 3 & & & - & 0.84 y $0.70(28)$ & 0.26 y $0.38(15)$ \\
\hline Sitio 4 & & & & - & 0.52 y $0.41(17)$ \\
\hline
\end{tabular}

0: no hay semejanza; 1 : son idénticos.

contraste, el sitio 5 registró similitudes muy bajas con los otros sitios, en particular con el sitio 1 (tabla 2). Patrones similares ocurren con el índice de Jaccard.

\section{Estructura de la comunidad}

Se registraron 1,183 individuos. La altura total promedio de las especies del pastizal fue de $19.5 \pm 0.05 \mathrm{~cm}$ y la cobertura total promedio de $12.98 \pm 0.83 \mathrm{~cm}$. El pastizal está formado por una carpeta continua de vegetación que cubre el $98 \%$ de la superficie. A pesar de una cierta homogeneidad general, fue posible distinguir 4 estratos (rasante, bajo, intermedio y alto) de acuerdo con la altura de las especies (tabla 3). Los estratos rasante y bajo albergan 11 especies cada uno y contribuyen con el 13.7 y $15.7 \%$ de la cobertura, respectivamente; el estrato intermedio presentó 24 especies y aportó el $46.38 \%$ de la cobertura; finalmente el estrato alto incluye 9 especies y $24.16 \%$ de la cobertura.

\section{Valores de importancia relativa}

De las 55 especies registradas durante el análisis estructural, 4 de ellas mostraron un VIR mayor al 5\%. Las especies dominantes en el pastizal son: M. phalaroides, Schizachyrium sanguineum, Sporobolus indicus y Pennisetum clandestinum (tabla 4), en gran medida por su cobertura relativa; estas 4 especies aportan cerca del $32 \%$ de la suma del valor de importancia.

La mayoría de las especies presentes en el pastizal se clasificaron en las categorías de dominantes y raras. En la categoría de especies dominantes se registraron 27 de estas, aunque P. clandestinum, Cyperus sp., Polygala alba y Macroptilium gibbosifolium, están colocadas en el límite entre dominantes e indicadoras. Entre las especies comunes solo se observó a
Plantago australis y Tinantia erecta; no obstante, estas también tuvieron una posición marginal entre las comunes y las raras. En la condición de especies raras se localizaron 23 taxa. Finalmente, en la categoría de especies indicadoras únicamente quedaron Agrostis hyemalis, Eragrostis tenuifolia y Verbena bipinnatifida (fig. 2).

\section{Discusión}

La riqueza y la composición de especies observadas en el área estudiada evidencian que el pastizal tiene potencial para la conservación de la biodiversidad. Merece una mención especial el hallazgo de una nueva especie de orquídea, la cual se ubica en el género Cyclopogon; esto confirma la necesidad de explorar botánicamente el territorio del Estado de México de manera intensa, pues a pesar de contar con un listado de su flora (Martínez y Matuda, 1979), esta deberá actualizarse.

La riqueza específica del pastizal es mayor que la reportada para un pastizal de Bouteloua dactyloides (Cruz, 1969), la consignada para la vegetación arvense de áreas de cultivo del Valle de Toluca (Vieyra-Odilón y Vibrans, 2001) y un campo de cultivo abandonado (> 25 años; González-Plata, 1998), donde se registraron 35, 74 y 85 especies en cada estudio; y menor que la obtenida para un pastizal de Querétaro (Suárez-Martínez, 1999), un pastizal de Hilaria cenchroides del Valle de México (Cruz, 1969) y claros de bosque (pino-encino), caminos y alrededor de cuerpos de agua (pozas y una presa; Rivas-Manzano y Vibrans, 2000) en los cuales se registraron 141, 233 y 300 especies en cada investigación. Otros pastizales evaluados asociados a los bosques templados de la cuenca de México (SilvaPérez, 1998; Velázquez-Montes y Romero, 1998), muestran una baja diversidad y difieren significativamente en la composición 
Tabla 3

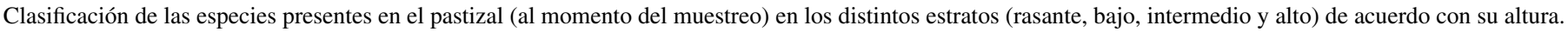

\begin{tabular}{|c|c|c|c|}
\hline Rasante $(1-5 \mathrm{~cm})$ & Bajo $(>5-10 \mathrm{~cm})$ & Intermedio $(>10-30 \mathrm{~cm})$ & Alto $(>30-50 \mathrm{~cm})$ \\
\hline Bulbostylis capillaris & Castilleja moranensis & Bidens anthemoides & Agrostis hyemalis \\
\hline Echeandia nana & Cologania obovata & Conyza sophiifolia & Bouteloua gracilis \\
\hline Eleocharis sp. & Commelina dianthifolia & Cyperus manimae & Chloris submutica \\
\hline Euphorbia nutans & Drymaria glandulosa & Cyperus sp. & Eragrostis tenuifolia \\
\hline Evolvulus prostratus & Erigeron pubescens & Diastatea sp. & Muhlenbergia montana \\
\hline Galium microphyllum & Eryngium carlinae & Digitaria ischaemum & Muhlenbergia rigida \\
\hline Heliopsis аппиа & Pennisetum clandestinum & Eryngium serratum & Schizachyrium sanguineum \\
\hline Macroptilium gibbosifolium & Tinantia erecta & Hilaria belangeri & Schkuhria pinnata var. virgata \\
\hline Matelea decumbens & Tradescantia crassifolia var. acaulis & Hilaria cenchroides & Sporobolus indicus \\
\hline Dioscorea multinervis & Trifolium pratense & Juncus sp. & \\
\hline \multirow[t]{14}{*}{ Polygala alba } & Viola grahamii & Kyllinga pumila & \\
\hline & & Luzula caricina & \\
\hline & & Muhlenbergia phalaroides & \\
\hline & & Plantago australis & \\
\hline & & Plantago lanceolata & \\
\hline & & Роа аппиа & \\
\hline & & Pseudognaphalium stramineum & \\
\hline & & Salvia reptans & \\
\hline & & Setaria parviflora & \\
\hline & & Steinchisma cupreum & \\
\hline & & Stevia eupatoria & \\
\hline & & Tagetes lucida & \\
\hline & & Tagetes micrantha & \\
\hline & & Verbena bipinnatifida & \\
\hline
\end{tabular}

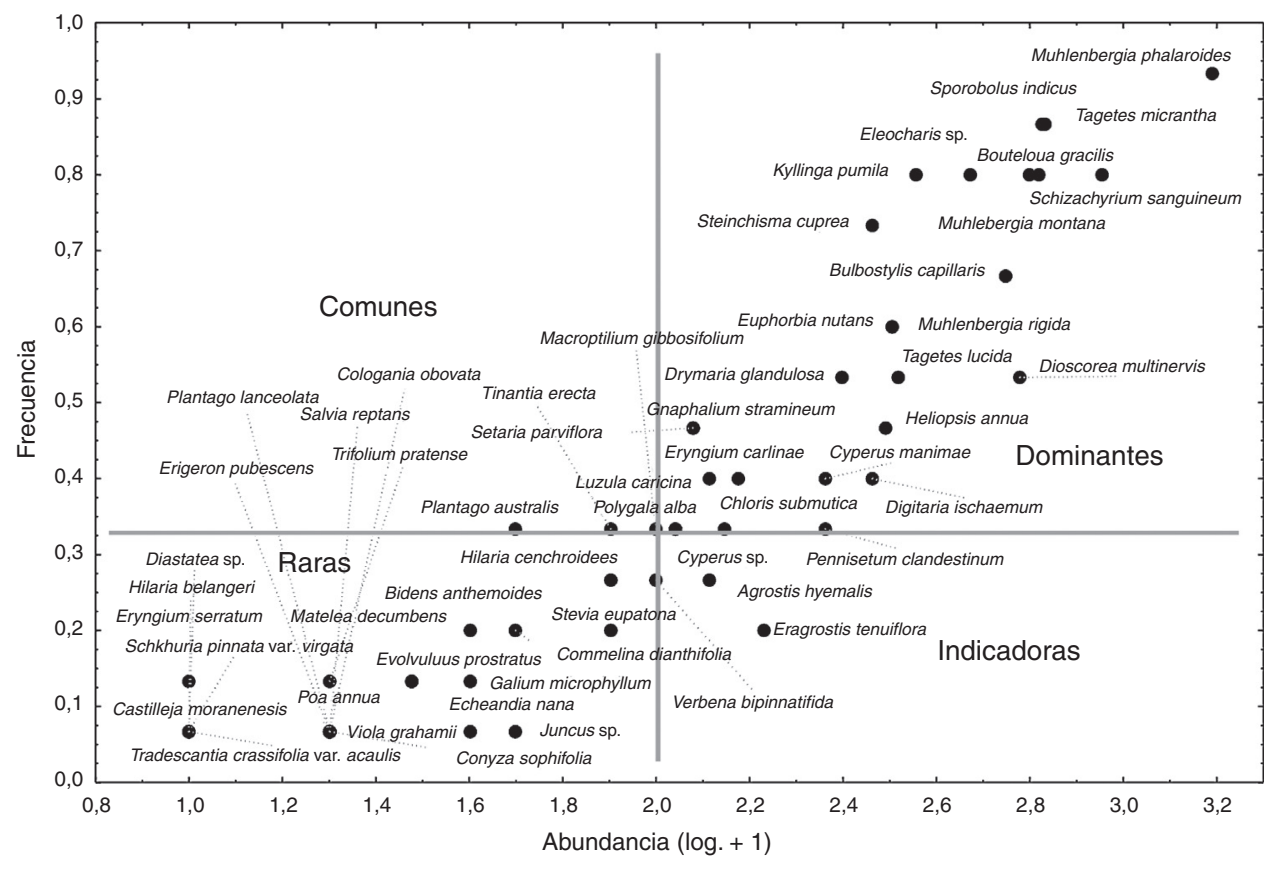

Figura 2. Clasificación de las especies registradas en el pastizal como dominantes, comunes, raras e indicadoras.

florística con este pastizal. Muchos de estos pastizales inducidos se encuentran inmersos en la vegetación natural correspondiente a bosques templados y otros en potreros establecidos en áreas que originalmente presentaban selva alta perennifolia; además de pastos nativos, en ellos es frecuente encontrar individuos adultos y plántulas de árboles remanentes de selva, lo que contribuye a su diversidad (Guevara, Meave, Moreno-Casasola, Laborde y Castillo, 1994; Lira-Noriega, Guevara, Laborde y
Sánchez-Ríos, 2007). La diversidad que presentan estas áreas se debe tanto a la migración de especies de la vegetación original como a la presión de alteración. En contraste, el pastizal estudiado se encuentra aislado de las áreas boscosas que pudieran mantener flujo de especies con este sitio y es evidente la ausencia de árboles y arbustos (fig. 1A). Aunque la presión de la alteración (incendios y pastoreo) es constante, prácticamente no se presenta suelo desnudo y la riqueza de especies 
Tabla 4

Densidad, frecuencia, cobertura (relativas) y valor de importancia relativa (VIR) de las especies analizadas en el pastizal.

\begin{tabular}{|c|c|c|c|c|}
\hline Especie & $\begin{array}{l}\text { Densidad relativa } \\
(\%)\end{array}$ & $\begin{array}{l}\text { Frecuencia relativa } \\
(\%)\end{array}$ & $\begin{array}{l}\text { Cobertura relativa } \\
(\%)\end{array}$ & $\begin{array}{l}\text { VIR } \\
(\%)\end{array}$ \\
\hline Muhlenbergia phalaroides & 13.1 & 4.83 & 25.9 & 14.6 \\
\hline Schizachyrium sanguineum & 7.61 & 4.14 & 6.14 & 5.97 \\
\hline Sporobolus indicus & 5.75 & 4.48 & 6.65 & 5.63 \\
\hline Pennisetum clandestinum & 1.94 & 1.72 & 12.2 & 5.3 \\
\hline Bouteloua gracilis & 5.33 & 4.14 & 4.67 & 4.7 \\
\hline Tagetes micrantha & 5.67 & 4.48 & 3.82 & 4.67 \\
\hline Muhlenbergia montana & 5.58 & 4.14 & 3.96 & 4.57 \\
\hline Eleocharis sp. & 3.97 & 4.14 & 3.05 & 3.73 \\
\hline Bulbostylis capillaris & 4.73 & 3.45 & 2.49 & 3.57 \\
\hline Dioscorea multinervis & 5.07 & 2.76 & 2.81 & 3.53 \\
\hline Tagetes lucida & 2.79 & 2.76 & 3.19 & 2.91 \\
\hline Steinchisma cupreum & 2.45 & 3.79 & 2.06 & 2.77 \\
\hline Kyllinga pumila & 3.04 & 4.14 & 1.05 & 2.74 \\
\hline Muhlenbergia rigida & 2.71 & 3.1 & 2.23 & 2.68 \\
\hline Euphorbia nutans & 2.71 & 3.1 & 1.39 & 2.4 \\
\hline Heliopsis аппиа & 2.62 & 2.41 & 2.02 & 2.35 \\
\hline Digitaria ischaemum & 2.45 & 2.07 & 2.29 & 2.27 \\
\hline Drymaria glandulosa & 2.11 & 2.76 & 1.6 & 2.16 \\
\hline Cyperus manimae & 1.94 & 2.07 & 0.68 & 1.56 \\
\hline Eryngium carlinae & 1.27 & 2.07 & 1.08 & 1.47 \\
\hline Luzula caricina & 1.1 & 2.07 & 0.98 & 1.38 \\
\hline Pseudognaphalium stramineum & 1.01 & 2.41 & 0.48 & 1.3 \\
\hline Setaria parviflora & 1.01 & 2.41 & 0.46 & 1.3 \\
\hline Chloris submutica & 1.18 & 1.72 & 0.66 & 1.19 \\
\hline Eragrostis tenuifolia & 1.44 & 1.03 & 1.07 & 1.18 \\
\hline Agrostis hyemalis & 1.11 & 1.38 & 0.89 & 1.12 \\
\hline Macroptilium gibbosifolium & 0.85 & 1.72 & 0.75 & 1.11 \\
\hline Polygala alba & 0.93 & 1.72 & 0.44 & 1.03 \\
\hline Cyperus sp. & 0.93 & 1.03 & 0.51 & 0.94 \\
\hline Verbena bipinnatifida & 0.85 & 1.38 & 0.59 & 0.94 \\
\hline Tinantia erecta & 0.68 & 1.72 & 0.27 & 0.89 \\
\hline Plantago australis & 0.42 & 1.72 & 0.25 & 0.8 \\
\hline Hilaria cenchroides & 0.68 & 1.38 & 0.29 & 0.78 \\
\hline Stevia eupatoria & 0.68 & 1.03 & 0.51 & 0.74 \\
\hline Bidens anthemoides & 0.34 & 1.03 & 0.34 & 0.57 \\
\hline Commelina dianthifolia & 0.42 & 1.03 & 0.15 & 0.54 \\
\hline Evolvulus prostratus & 0.25 & 0.69 & 0.23 & 0.39 \\
\hline Galium microphyllum & 0.34 & 0.69 & 0.14 & 0.39 \\
\hline Matelea decumbens & 0.25 & 0.69 & 0.22 & 0.39 \\
\hline Trifolium pratense & 0.17 & 0.69 & 0.05 & 0.3 \\
\hline Juncus $\mathrm{sp}$. & 0.42 & 0.34 & 0.12 & 0.29 \\
\hline Echeandia nana & 0.34 & 0.34 & 0.16 & 0.28 \\
\hline Eryngium serratum & 0.08 & 0.69 & 0.07 & 0.28 \\
\hline Роа аппиа & 0.17 & 0.34 & 0.23 & 0.25 \\
\hline Cologania obovata & 0.17 & 0.34 & 0.21 & 0.24 \\
\hline Salvia reptans & 0.17 & 0.34 & 0.12 & 0.21 \\
\hline Plantago lanceolata & 0.17 & 0.34 & 0.07 & 0.2 \\
\hline Castilleja moranensis & 0.17 & 0.34 & 0.05 & 0.19 \\
\hline Conyza sophiifolia & 0.17 & 0.34 & 0.07 & 0.19 \\
\hline Viola grahamii & 0.17 & 0.34 & 0.05 & 0.19 \\
\hline Erigeron pubescens & 0.17 & 0.34 & 0.03 & 0.18 \\
\hline Hilaria belangeri & 0.08 & 0.34 & 0.1 & 0.18 \\
\hline Schkuhria pinnata var. virgata & 0.08 & 0.34 & 0.05 & 0.16 \\
\hline Diastatea sp. & 0.08 & 0.34 & 0.03 & 0.15 \\
\hline Tradescantia crassifolia var. acaulis & 0.08 & 0.34 & 0.03 & 0.15 \\
\hline
\end{tabular}

es alta. El número de familias (40) y de géneros (93) registrados en la presente investigación es similar a los reportados en estos trabajos. Las familias Poaceae, Asteraceae y Fabaceae fueron las más diversas, en consistencia con estudios florísticos realizados no solo en el Estado de México sino en todo el país (Martínez y Matuda, 1979; Rzedowski, 1991; Villaseñor, 2003, 2004; Villaseñor y Ortiz, 2014). Sin embargo, estas mismas familias incluyeron la mayor riqueza de especies exóticas, en concordancia con el reporte de Villaseñor y Espinosa-García (2004). 
Tabla 5

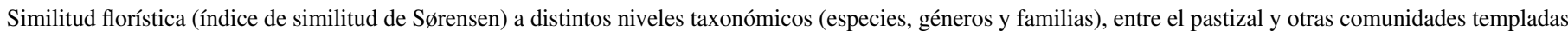
dominadas por gramíneas.

\begin{tabular}{|c|c|c|c|c|}
\hline \multirow[t]{2}{*}{ Nivel taxonómico } & \multicolumn{4}{|c|}{ Similitud de Sørensen } \\
\hline & $\mathrm{CCA}^{\mathrm{a}}$ & $\mathrm{CPP}^{\mathrm{b}}$ & $\mathrm{VA}^{\mathrm{c}}$ & $\mathrm{PQ}^{\mathrm{d}}$ \\
\hline Especies & $0.07(7)$ & $0.10(20)$ & $0.13(12)$ & $0.11(14)$ \\
\hline Géneros & $0.32(27)$ & $0.21(19)$ & $0.33(26)$ & $0.31(32)$ \\
\hline Familias & $0.54(20)$ & $0.30(15)$ & $0.47(15)$ & $0.53(21)$ \\
\hline
\end{tabular}

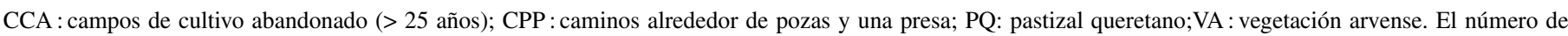
especies, géneros y familias en común se muestra entre paréntesis.

a González-Plata (1998).

b Rivas-Manzano y Vibrans (2000).

c Vieyra-Odilón y Vibrans (2001).

d Suárez-Martínez (1999).

La elevada riqueza de especies del pastizal refuerza el trabajo de Wilson et al. (2012), quienes mencionan que los pastizales junto con los bosques tropicales lluviosos son los 2 ecosistemas terrestres con mayor diversidad y que aun cuando los pastizales son ecosistemas sometidos a perturbaciones moderadas, estos pueden albergar una gran diversidad de especies en una superficie muy pequeña $\left(<100 \mathrm{~m}^{2}\right)$; sin embargo, esta elevada diversidad podría estar relacionada con la heterogeneidad ambiental (Collins y Barber, 1986) por lo que para planes de conservación y diseño de algunas reservas ecológicas habrán de ser tomados en cuenta.

La similitud florística entre el pastizal y otras comunidades de origen secundario dominadas por gramíneas a distintos niveles taxonómicos reveló que un 50\% de las familias se comparten entre las distintas comunidades; no obstante, esta similitud es menor a nivel genérico con $30 \%$ de los géneros compartidos y a nivel específico la similitud florística es todavía menor con $15 \%$ de las especies compartidas (tabla 5). Estas cifras ponen de manifiesto que el pastizal estudiado podría tener un origen distinto a las comunidades con las que fue comparado. Sin embargo, también es evidente el grado de alteración del pastizal, pues muchas de las especies compartidas entre el pastizal y las otras comunidades son ruderales o arvenses, presentes en pastizales secundarios, orilla de caminos, campos de cultivo y sitios perturbados de bosques de pino-encino. Finalmente, algunas otras plantas resultaron ser exóticas, como $P$. clandestinum, especie conocida por su gran capacidad invasora.

La alta incidencia de los disturbios antrópicos y la presencia de especies exóticas en el pastizal, principalmente gramíneas, permiten conjeturar que el pastizal podría tener un origen antrópico, derivado de bosques de pino-encino, como es el caso de muchos pastizales presentes en el Estado de México. Sin embargo, no se tiene información suficiente para dilucidar con precisión su origen; no obstante, cualquiera que este sea, no se puede hacer a un lado la posibilidad de que algunos pastizales templados del centro del país tengan un origen natural (primario), como se ha propuesto para algunos sistemas equivalentes localizados en las regiones tropicales del país (Hernández, 1964; López-Olmedo et al., 2007; Pérez-García y Meave, 2006). Por otra parte, la invasión de especies exóticas en estas comunidades podría ser demasiado rápida, de modo que estas logran desplazar a las nativas debido a su mayor capacidad competitiva. Para poder resolverlo, estudios futuros tendrán que evaluar la dinámica de las especies invasoras, así como la composición de especies en pastizales con exclusión de pastoreo y sin fuego, para establecer su posible origen.

La comparación de los índices de diversidad calculados en este trabajo con un estudio realizado por López-Olmedo et al. (2006) para una sabana de origen primario en la región del sureste del país es similar (3.36 vs. 3.64), aun cuando estos difieren sustancialmente en los métodos de muestreo y condiciones climáticas. Por otra parte, las diferencias entre los diferentes sitios analizados a lo largo de la pendiente pueden estar relacionadas tanto con la abundancia y riqueza de especies, como con efectos diferenciales de los disturbios antrópicos, o incluso con las condiciones microclimáticas donde se realizaron los censos de la vegetación, las cuales podrían estar condicionando la distribución de la especies por algún factor como la variación en la humedad del suelo (Álvarez-Lopeztello, 2010). La similitud florística entre los sitios intermedios (2, 3 y 4), y la disimilitud con los extremos (1 y 5), también apoyan esta posibilidad (tabla 2).

En general, la cobertura del pastizal se extiende sobre casi toda la superficie (98\%) del terreno (fig. 1B), lo que sugiere que los disturbios aún no ejercen gran presión sobre la vegetación; sin embargo, esto no es muy claro del todo pues, si se llegase a ejercer se están seleccionando especies de porte intermedio y bajo (tabla 3) que prosperan bajo estas circunstancias. Poaceae fue estructuralmente la familia dominante en el pastizal seguida de Asteraceae y Cyperaceae con más de la mitad de la suma de valores de importancia de las especies del pastizal. Por otra parte, el análisis estructural reveló que $M$. phalaroides, $S$. sanguineum, $S$. indicus y $P$. clandestinum son las especies dominantes y con el mayor VIR. Es probable que esta dominancia esté relacionada con el hecho de que dichas especies puedan estar adaptadas en cierto grado al fuego y pastoreo. Es importante señalar que aun cuando $P$. clandestinum está presente, no es la especies más dominante, una posible explicación a esto puede ser su introducción al pastizal en años recientes, por lo tanto aun no ejerce gran capacidad competitiva, con el resto de las especies dominantes a pesar de su amplio rango de tolerancia a condiciones ambientales (Muscolo, Panuccio y Sidari, 2003).

Con respecto a las especies dominantes, comunes, raras e indicadoras en el pastizal, la mayoría de los taxa dominantes pertenecen a las familias Poaceae y Cyperaceae, como es de 
esperarse para este tipo de vegetación. Además, un gran número de especies se clasificaron en la categoría de raras, lo que pone en evidencia que si bien algunas especies de gramíneas son favorecidas por el fuego y el pastoreo, muchas otras pueden no tolerar estos factores y por lo tanto podrían desaparecer de la comunidad solo con un incremento en la frecuencia de dichos factores.

En síntesis los resultados de este estudio exhiben la necesidad de acrecentar las investigaciones en pastizales templados y de sus equivalentes en las zonas tropicales del territorio nacional. Además, se requieren estudios experimentales a largo plazo con exclusión de fuego y pastoreo en estos ecosistemas, para dilucidar con precisión la naturaleza de muchas de estas comunidades vegetales, aparentemente de origen secundario, con la finalidad de sugerir estrategias adecuadas de conservación, manejo, aprovechamiento racional y restauración.

\section{Agradecimientos}

Agradecemos a Hilda Flores Olvera y a Blanca Verónica Juárez Jaimes por facilitarnos el acceso a las instalaciones del Herbario Nacional (MEXU) de la Universidad Nacional Autónoma de México para el cotejo de ejemplares; a Gerardo A. Salazar Chávez y Rafael Torres Colín por su ayuda en la determinación taxonómica de algunos ejemplares de las familias Orchidaceae y Dioscoreaceae. Jesús Enrique Soto Ramos facilitó los datos de temperatura y precipitación y Guillermo Enríquez Marín colaboró en el trabajo de campo. Ednita Espinoza Hernández y Ana Mejía Canales facilitaron las imágenes. Finalmente, agradecemos a Jorge A. Meave y José Luis Álvarez Lopeztello, quienes leyeron el manuscrito y contribuyeron con valiosas críticas y sugerencias a fin de mejorar su calidad.

\section{Apéndice. Listado de especies encontradas en el pastizal. El asterisco $\left(^{*}\right)$ indica las especies introducidas a México}

\begin{tabular}{|c|c|}
\hline Familias y especies & Familia y especies \\
\hline Monocotiledoneas & Schiedeella llaveana var. alinae Szlach. \\
\hline Asparagaceae & Schiedeella tenella (L.O. Williams) Burns-Bal. \\
\hline Echeandia flavescens (Schult y Schult.) Cruden & Poaceae \\
\hline Echeandia nana (Baker) Cruden & Agrostis hyemalis (Walter) Britton, Sterns y Poggenb.*5 \\
\hline Commelinaceae & Bouteloua gracilis (Kunth) Lag. ex Griffiths \\
\hline Commelina tuberosa $\mathrm{L}$. & Bouteloua simplex Lag. \\
\hline Tinantia erecta (Jacq.) Schltdl. & Chascolytrum subaristatum (Lam.) Desv. \\
\hline Tradescantia crassifolia var. acaulis (M. Martens y Galeotti) C.B. Clarke & Chloris submutica Kunth \\
\hline Cyperaceae & Cynodon dactylon (L.) Pers.* 1 \\
\hline Bulbostylis capillaris (L.) Kunth ex C.B. Clarke & Digitaria ischaemum (Schreb.) Muhl.* 1 \\
\hline Cyperus esculentus L. * 2 & Echinochloa oplismenoides (E. Fourn.) Hitchc. \\
\hline Cyperus manimae Kunth & Eleusine multiflora Hochst. ex A. Rich.* 1 \\
\hline Cyperus sp. & Eragrostis intermedia Hitchc. \\
\hline Eleocharis elegans (Kunth) Roem y Schult. & Eragrostis $\mathrm{sp}$. \\
\hline Eleocharis sp. & Eragrostis tenuifolia (A. Rich.) Hochst. ex Steud.* 1 \\
\hline Kyllinga pumila Michx. & Hilaria belangeri (Steud.) Nash \\
\hline Dioscoreaceae & Hilaria cenchroides Kunth \\
\hline Dioscorea multinervis Benth. & Muhlenbergia montana (Nutt.) Hitchc. \\
\hline Hypoxidaceae & Muhlenbergia phalaroides (Kunth) P.M. Peterson \\
\hline Hypoxis mexicana Schult y Schult. f. & Muhlenbergia rigida (Kunth) Kunth \\
\hline Iridaceae & Muhlenbergia tricholepis (Torr.) Columbus \\
\hline Nemastylis tenuis (Herb.) S. Watson & Pennisetum clandestinum Hochst. ex Chiov.* 1 \\
\hline Sisyrinchium scabrum Cham. y Schltdl. & Peyritschia pringlei (Scribn.) S.D. Koch \\
\hline Sisyrinchium tenuifolium Humb. y Bonpl. ex Willd. & Роа аппиа L.* 4 \\
\hline Sisyrinchium tolucense Peyr. & Schizachyrium sanguineum (Retz.) Alston \\
\hline Juncaceae & Setaria parviflora (Poir.) Kerguélen \\
\hline Juncus sp. & Sporobolus indicus (L.) R. Br. \\
\hline Juncus sp. & Steinchisma cupreum (Hitchc y Chase) W.V. Br. \\
\hline Luzula caricina E. Mey. & Pontederiaceae \\
\hline Lilaeaceae & Eichhornia crassipes (Mart.) Solms* 4 \\
\hline Lilaea scilloides (Poir.) Hauman & Eudicotiledoneas \\
\hline Liliaceae & Apiaceae \\
\hline Calochortus barbatus (Kunth) J.H. Painter & Eryngium carlinae F. Delaroche \\
\hline Orchidaceae & Eryngium serratum Cav. \\
\hline Brachystele affinis (C. Schweinf.) Burns-Bal y R. González & Tauschia nudicaulis Schltdl. \\
\hline Cyclopogon sp. nov. & Apocynaceae \\
\hline Sarcoglottis schaffneri (Rchb. f.) Ames & Asclepias glaucescens Kunth \\
\hline Apocynaceae & Gentianaceae \\
\hline Asclepias rzedowskii W.D. Stevens & Gentiana bicuspidata (G. Don.) Briq. \\
\hline Matelea decumbens W.D. Stevens & Hypericaceae \\
\hline Araliaceae & Hypericum formosum Kunth \\
\hline Hydrocotyle ranunculoides L.f. & Lamiaceae \\
\hline Asteraceae & Prunella vulgaris L. \\
\hline Bidens anthemoides (DC.) Sherff & Salvia leucantha Cav. \\
\hline
\end{tabular}




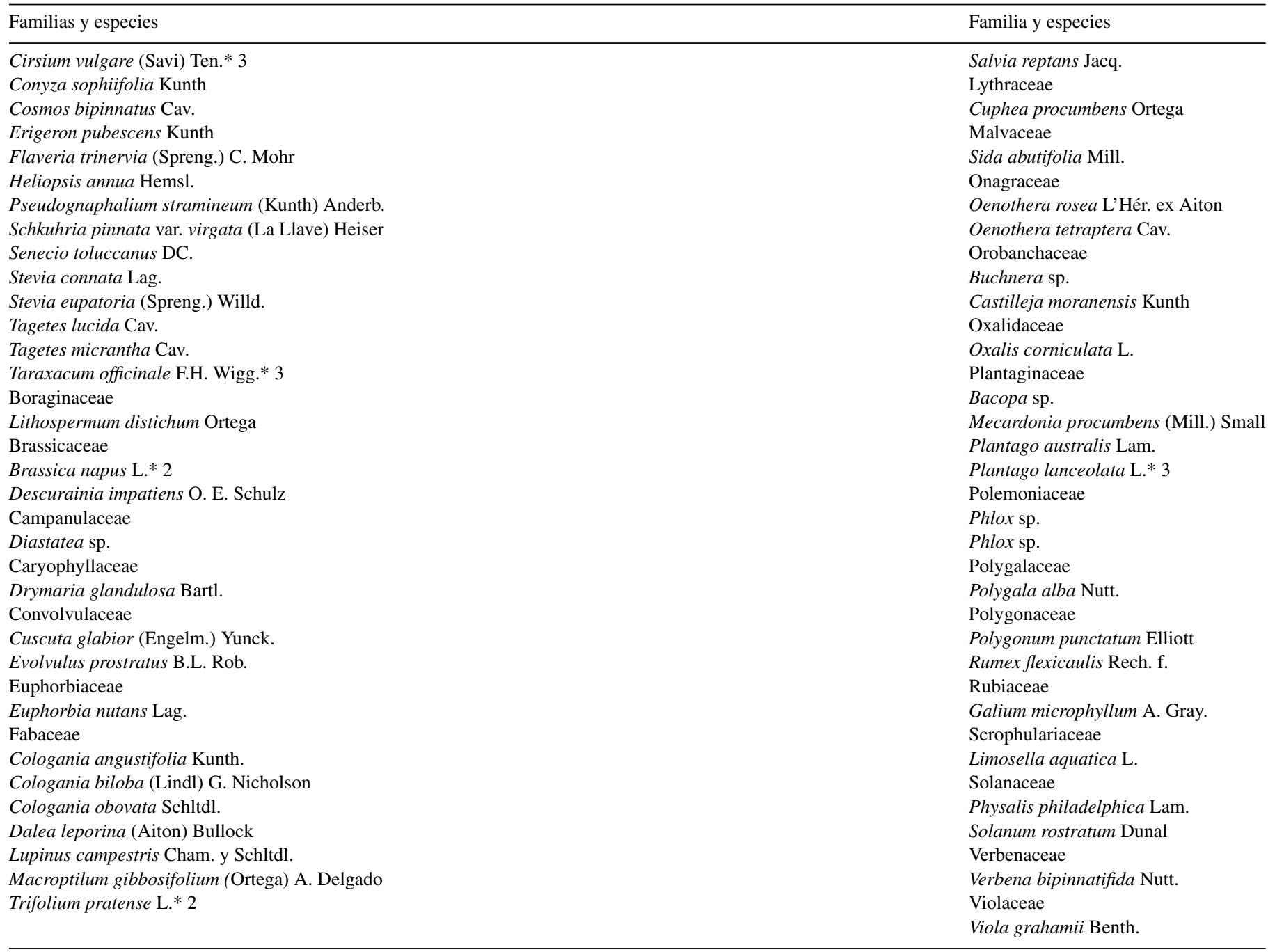

Origen de las especies introducidas: 1 = África; 2 =Europa; 3 = Eurasia; 4 = Sudamérica; 5 = Origen incierto.

\section{Referencias}

Álvarez-Lopeztello, J. (2010). Caracterización de un pastizal en «El Cerrillo Piedras Blancas», Toluca, Estado de México. Tesis. Facultad de Ciencias, Universidad Autónoma del Estado de México. Toluca, Estado de México.

Canfield, R. H. (1941). Applications of the line interception method in sampling range vegetation. Journal of Forestry, 39, 384-388.

Challenger, A. (1998). Utilización y conservación de los ecosistemas terrestres de México. Pasado presente y futuro. México, D.F.: Conabio: Universidad Nacional Autónoma de México y Agrupación Sierra Madre.

Collins, S. L. y Barber, S. C. (1986). Effects of disturbance on diversity in mixed-grass prairie. Vegetatio, 64, 87-94.

Conabio (Comisión nacional para el conocimiento y uso de la biodiversidad). (1998). La diversidad biológica de México: estudio de País. México: Conabio.

Coupland, R. T. (Ed.). (1979). Grassland ecosystems of the world: analysis of grasslands and their uses.. London: Cambridge University Press.

Cruz, C. R. (1969). Contribución al conocimiento de la ecología de los pastizales en el Valle de México. Tesis. Escuela Nacional de Ciencias Biológicas. Instituto Politécnico Nacional. México, D.F.

Dávila, P., Mejía-Saules, M. T., Gómez-Sánchez, M., Valdés-Reyna, J., Ortiz, J. J., Morín, C., et al. (2006). Catálogo de gramíneas de México. México, D.F: Universidad Nacional Autónoma de México y Conabio.
Espinosa-García, F. J. y Sarukhán, J. (1997). Manual de malezas del valle de México: claves, descripciones e ilustraciones. México, D.F: Universidad Nacional Autónoma de México y Fondo de Cultura Económica.

García, E. (1973). Modificaciones al sistema de clasificación climática de Köppen. Instituto de Geografía. México, D.F: Universidad Autónoma de México.

Gentry, H. S. (1957). Los pastizales de Durango, estudio ecológico, fisiográfico y florístico. México, D.F: Instituto Mexicano de Recursos Naturales Renovables.

González-Plata, M. (1998). Efecto de la perturbación sobre la dinámica de la vegetación en bosques de pino-encino en San Cayetano, Estado de México. Tesis. Facultad de Ciencias, Universidad Autónoma del Estado de México. Toluca, Estado de México.

Guevara, S., Meave, J., Moreno-Casasola, P., Laborde, J. y Castillo, S. (1994). Vegetación y flora de potreros en la Sierra de los Tuxtlas, México. Acta Botanica Méxicana, 28, 1-27.

Harrison, S., Inouye, B. D. y Safford, H. D. (2003). Ecological heterogeneity in the effects of grazing and fire on grassland diversity. Conservation Biology, $17,837-845$.

Hernández, E. (1964). Los pastos y pastizales de las zonas áridas del centro y noreste de México. En E. En y Beltrán (Eds.), Las zonas áridas del centro y noreste de México (pp. 97-127). México, D.F: Instituto Mexicano de Recursos Naturales Renovables. 
Hernández, E., Tapia, C. y Buller, R. (1957). Los pastizales del noreste. Agricultura Técnica en México, 1, 6-43.

Hobbs, R. J. y Huenneke, L. F. (1992). Disturbance, diversity and invasion: implications for conservation. Conservation Biology, 6, 324-337.

Lira-Noriega, A., Guevara, S., Laborde, J. y Sánchez-Ríos, G. (2007). Composición florística en potreros de los Tuxtlas, Veracruz, México. Acta Botanica Mexicana, 80, 59-87.

López-Olmedo, L. I., Pérez-García, E. A. y Meave, J. A. (2006). Estructura y composición florística de las sabanas de la región de Nizanda, Istmo de Tehuantepec (Oaxaca), México. Acta Botanica Mexicana, 77, $41-67$.

López-Olmedo, L. I., Meave, J. A. y Pérez-García, E. A. (2007). Floristic and structural contrasts between natural savannas and anthropogenic pastures in a tropical dry landscape. The Rangeland Journal, 29, 181-190.

Martínez, A. G. y Hernández, C. (2000). Monografía Delegación de Tlachaloya, Toluca. Toluca, Estado de México: H. Ayuntamiento de Toluca.

Martínez, M. y Matuda, E. (1979). Flora del Estado de México. Volúmenes I, II y III. In Biblioteca Enciclopédica del Estado de México. Toluca, Estado de México: Gobierno del Estado de México.

Miranda, F. y Hernández, E. (1963). Los tipos de vegetación de México y su clasificación. Boletín de la Sociedad Botánica de México, 28, 29-179.

Moreno, C. E. (2001). Métodos para medir la biodiversidad. In M\&T-Manuales y Tesis, Sociedad Entomológica Aragonesa. España: Zaragoza.

Muscolo, A., Panuccio, M. R. y Sidari, M. (2003). Effects of salinity on growth, carbohydrate metabolism and nutritive properties of kikuyu grass (Pennisetum clandestinum Hochst). Plant Science, 164, 1103-1110.

Pérez-García, E. A. y Meave, J. A. (2006). Coexistence and divergence of tropical dry forests and savannas in southern Mexico. Journal of Biogeography, 33, 438-447.

Reyes, C. R. y Zamora, S. C. (1977). Estudio de una sabana de montaña localizada en las estribaciones de la Sierra Madre del Sur. Ciencia Forestal, 2, $36-64$.

Rivas-Manzano, I. V. (1984). Estudios experimentales sobre la sucesión secundaria en agostaderos de «El Gran Tunal» San Luis Potosí. Tesis. México, D.F: Universidad Nacional Autónoma de México.

Rivas-Manzano, I. V. (1988). Cambios en el vecindario de Bouteloua gracilis por la exclusión del pastoreo en «El Gran Tunal», San Luis Potosí. Tesis de maestría. Colegio de Posgraduados, Chapingo, México.
Rivas-Manzano, I. V. y Vibrans, H. (2000). Composición florística de la unidad de San Cayetano, Villa de Allende, asociada a sitios con distinto tipo de perturbación. Informe final de investigación. Toluca, Estado de México: Facultad de Ciencias, Universidad Autónoma del Estado de México.

Rzedowski, J. (1975). An ecological and phytogeographical analysis of the grasslands of Mexico. Taxon, 24, 67-80.

Rzedowski, J. (1978). Vegetación de México. México, D.F: Limusa.

Rzedowski, J. (1991). Diversidad y orígenes de la flora fanerogámica de México. Acta Botanica Mexicana, 14, 3-21.

Rzedowski, J. y Calderón, G. (1990). Nota sobre el elemento africano en la flora adventicia de México. Acta Botanica Mexicana, 12, 21-24.

Rzedowski, G. C. y Rzedowski, J. (2005). Flora fanerogámica del Valle de México. México, D.F: Conabio - Instituto de Ecología, A.C.

Silva-Pérez, L. C. (1998). Los bosques de coníferas del sur de la cuenca de México: fitosociología, diversidad y uso tradicional. Tesis. Facultad de Ciencias, Universidad Autónoma del Estado de México, Toluca, Estado de México.

Sokal, R. y Rohlf, F. J. (1979). Biometría principios y métodos estadísticos en la investigación biológica. Madrid: Blume.

Suárez-Martínez, A. L. (1999). Diversidad florística de un pastizal en el estado de Querétaro. Tesis. Universidad Autónoma de Querétaro. Querétaro, Qro.

Velázquez-Montes, J. A. y Romero, F. J. (1998). Análisis de la heterogeneidad ambiental y conectividad de las Áreas Naturales del sur del Valle de México. México, D.F.: Facultad de Ciencias, Universidad Nacional Autónoma de México. Informe Final SNIB-Conabio Proyecto No. B144.

Vieyra-Odilón, L. y Vibrans, H. (2001). Weeds as crops: the value of maize field weeds in the valley of Toluca, Mexico. Economic Botany, 55, 426-443.

Villaseñor, J. L. (2003). Diversidad y distribución de las Magnoliophyta de México. Interciencia, 28, 160-167.

Villaseñor, J. L. (2004). Los géneros de plantas vasculares de la flora de México. Boletín de la Sociedad Botánica de México, 75, 105-135.

Villaseñor, J. L. y Espinosa-García, F. (2004). The alien flowering plants of Mexico. Diversity and Distributions, 10, 113-123.

Villaseñor, J. L. y Ortiz, E. (2014). Biodiversidad de las plantas con flores (División Magnoliophyta) en México. Revista Mexicana de Biodiversidad, Supl., 85, 134-142.

Wilson, J. B., Peet, R. K., Dengler, J. y Pärtel, M. (2012). Plant species richness: the world records. Journal of Vegetation Science, 23, 792-802. 